\title{
The Analysis of Fruit Protection Mechanisms Provided by Reduced-Pathogenicity Mutants of Colletotrichum gloeosporioides Obtained by Restriction Enzyme Mediated Integration
}

\author{
N. Yakoby, D. Beno-Moualem, I. Kobiler, and D. Prusky
}

Department of Postharvest Science of Fresh Produce, Agricultural Research Organization, The Volcani Center, Bet Dagan 50250, Israel. Current address of N. Yakoby: The BioTech Center, Rutgers University, Cook College, New Brunswick, NJ 08901. Accepted for publication 17 June 2002.

\begin{abstract}
Yakoby, N., Beno-Moualem, D., Kobiler, I., and Prusky, D. 2002. The analysis of fruit protection mechanisms provided by reduced-pathogenicity mutants of Colletotrichum gloeosporioides obtained by restriction enzyme mediated integration. Phytopathology 92:1196-1201.

Colletotrichum gloeosporioides is an important postharvest pathogen that attacks ripe avocado fruit. Two reduced-pathogenicity mutants, $\mathrm{Cg}$ M-142 and Cg-M-1150, previously obtained by restriction enzyme mediated integration, were used for the sequential analysis of the induction of biocontrol in avocado fruit. Plant biochemical indicators, such as $\mathrm{H}^{+}-$

Cg-M-142 enhanced $\mathrm{H}^{+}$-ATPase activity and the production of reactive oxygen species. These early signaling events were followed by higher phenylalanine ammonia lyase activity and higher levels of epicatechin and the antifungal diene, and decay was delayed. Unlike $\mathrm{Cg}-\mathrm{M}-142, \mathrm{Cg}-$ M-1150 did not activate early signaling events related to fruit resistance. We suggest that the initiation of early signaling events affecting fruit resistance is determined by the capability of the pathogen to interact with the fruit during appressorium formation. Furthermore, the intensity of the fruit defense response determines the level of resistance during fruit storage.
\end{abstract} ATPase activity and levels of reactive oxygen species, phenylalanine ammonia lyase, epicatechin, and an antifungal diene, were investigated. The main difference between Cg-M-142 and Cg-M-1150 was the lack of appressorium formation by the latter. Preinoculation of avocado fruit with
Additional keywords: fruit signal transduction, pathogen-fruit interaction, postharvest decay.
The phytopathogenic fungus Colletotrichum gloeosporioides (Penz.) Penz. \& Sacc. (teleomorph Glomerella cingulata) is an important postharvest pathogen attacking a wide range of tropical and subtropical fruits $(8,25)$. C. gloeosporioides initiates its attack by conidium germination and appressorium formation (5), followed by the production of infection hyphae, which penetrate the epidermal cells of the avocado exocarp. The hyphae remain quiescent until the fruit ripens and softens during storage (19). It has been hypothesized (19) that several mechanisms are responsible for the resistance of unripe fruit to fungal attack during quiescence: (i) the lack of nutrient supply for the pathogen in unripe fruit, (ii) the lack of secretion of fungal pathogenicity factors, (iii) the presence of preformed antifungal compounds, and (iv) the presence of inducible antifungal compounds. The last two mechanisms have been suggested as the main factors affecting avocado resistance (19).

The level of 1-acetoxy-2-hydroxy-4-oxo-heneicosa-12,15-diene (antifungal diene) depends on the synthesis and metabolism of the compound (24). The synthesis of diene includes the activation of $\Delta^{9}$ desaturase (L. Madi, W. Xuejun, I. Kobiler, A. Lichter, and D. Prusky, unpublished data), $\Delta^{12}$ desaturase (W. Xuejun et al., unpublished data), and a fatty acid elongase (Xuejun et al., unpublished data). Transcriptional activation of the two genes has been observed in avocado as a result of inoculation with C. gloeosporioides (Xuejun et al., unpublished data). The metabolism of the antifungal diene has been attributed to the oxidative activity of lipoxygenase (23). The activity of lipoxygenase is modulated by the level of flavan-3-ol-epicatechin (epicatechin) (12), a natural

Corresponding author: D. Prusky; E-mail address: dovprusk@agri.gov.il

Publication no. P-2002-0903-01R

(c) 2002 The American Phytopathological Society antioxidant phenol generated in the phenylpropanoid pathway and present in the avocado exocarp. The level of epicatechin declines during fruit ripening, and as a result lipoxygenase can reduce diene levels (24). Transcriptional activation of genes encoding epicatechin-forming proteins in the phenylpropanoid pathway, such as phenylalanine ammonia lyase (PAL), has been shown to increase diene and concomitant fruit resistance $(2,4,27)$. It is still unclear what early events activate the mechanism of resistance in avocado fruit. It was recently reported that the production of reactive oxygen species (ROS) in avocado significantly increased after inoculation with $C$. gloeosporioides conidia (4). It has been suggested that quiescent infection may be a local source of ROS production. This increase in ROS activates the phenylpropanoid pathway, which subsequently increases the level of antifungal diene and enhances fungal inhibition.

Recently, we generated reduced-pathogenicity mutants of $C$. gloeosporioides on avocado fruit, by insertional mutagenesis using restriction enzyme mediated integration (REMI) technology (36). Isolate Cg-M-142 produced appressoria on the exocarp, like the wild-type isolate $\mathrm{Cg}-14$, but it caused late and minor symptom development in ripening fruit. Isolate $\mathrm{Cg}-\mathrm{M}-1150$ did not produce appressoria on the exocarp, nor did it cause any symptoms. Preinoculation of avocado fruit with $\mathrm{Cg}-\mathrm{M}-142$ delayed the development of symptoms caused by the wild-type isolate and enhanced diene levels (36). Preinoculation with Cg-M-1150 did not affect the level of diene, nor did it delay the appearance of decay symptoms caused by $\mathrm{Cg}-14$. The present work describes the interaction of the reduced-pathogenicity mutant and avocado, with appressorium formation as a main factor in early events (4) leading to the activation of a mechanism of resistance to Colletotrichum. We propose a model to explain the recognition of the fungus by avocado fruit and the importance of appressorium formation as a factor in host-plant interactions. 


\section{MATERIALS AND METHODS}

Avocado fruit, fungal isolate, and growth media. Experiments were carried out with freshly harvested fruit of the avocado cultivar Fuerte (Persea americana Mill. var. drymifolia (Schltdl. \& Cham.) S. F. Blake) from an orchard at Kibbutz Givat Brenner, Israel. A single-conidium isolate of C. gloeosporioides, Cg-14, was obtained from a decayed fruit of the avocado cultivar Fuerte, and conidia were maintained in $10 \mathrm{mM}$ Na-phosphate buffer $(\mathrm{pH}$ 7.2), $40 \%$ glycerol, at $-80^{\circ} \mathrm{C}$. Three-week-old conidia were harvested from Mathur's medium $\left(\mathrm{M}_{3} \mathrm{~S}\right)$ plates (3), counted with the aid of a hemacytometer (Brand, Wertheim, Germany), and used for culture and fruit inoculation. Tissue treatment and inoculation were performed as previously described (4). Experiments were conducted in two consecutive harvesting seasons and repeated at least three times per season. The data presented are usually the average values of three to five replications of a single experiment. In some cases the average of all the replications of repeated experiments are presented with their pooled standard error.

Fruit protection assays and phenotypic characterization of transformed isolates. Twenty freshly harvested avocado fruits (cv. Fuerte) were dipped for $30 \mathrm{~s}$ in suspensions of conidia $(5 \times$ $10^{5}$ conidia/ml) of the two C. gloeosporioides mutants (Cg-M-142 and $\mathrm{Cg}-\mathrm{M}-1150)$ and then stored at $90 \%$ relative humidity and $20^{\circ} \mathrm{C}$ for $24 \mathrm{~h}$. Thereafter, the treated fruit was spot-inoculated with a $\mathrm{Cg}-14$ conidial suspension $\left(1 \times 10^{6}\right.$ conidia/ml $)(36)$. Appressoria were observed under a light microscope (BH-2 Olympus, Tokyo, Japan) in a 0.5-mm-deep slice of inoculated exocarp cut out with a scalpel 24 and $48 \mathrm{~h}$ after inoculation. Isolate growth rates were measured in five replicates on exocarp and on glass slides by inoculation with $10 \mu \mathrm{l}$ of the conidial suspension described above. All experiments were repeated at least three times over two consecutive avocado seasons. Exocarp inoculation was carried out by brushing exocarp strips with a conidial suspension $\left(2 \times 10^{6} \mathrm{conidia} / \mathrm{ml}\right)(22)$. As a control, exocarp strips were brushed with distilled water only.

Extraction of epicatechin. Epicatechin was extracted from $3 \mathrm{~g}$ of 1-mm-thick avocado exocarp strips, as previously described (21). Epicatechin was quantified in a reverse-phase high-performance liquid chromatography (HPLC) (RP-18) column eluted with $55 \%$ methanol and $1 \%$ acetic acid in double-distilled water. Calculation of the epicatechin level was based on comparison of the HPLC peak areas quantified at $275 \mathrm{~nm}$ with those of the standard, (2R,3S)-2-(3,4-dihydroxyphenyl)-3,4-dihydro-1(2H)-benzopyran3,5,7-triol (Sigma, Jerusalem, Israel).

Extraction of PAL and analysis of PAL activity. Samples for analysis of PAL activity were extracted by blending $5 \mathrm{~g}$ of avocado exocarp in $50 \mathrm{ml}$ of cold acetone $\left(-20^{\circ} \mathrm{C}\right)$ and collecting the homogenate on Whatman no. 1 filter paper. This process was carried out four times. The exocarp powder was dried at room temperature overnight and stored at $-20^{\circ} \mathrm{C}$ until used. PAL was extracted from $100 \mathrm{mg}$ of exocarp powder stirred for $2 \mathrm{~h}$ at $4^{\circ} \mathrm{C}$ with $5 \mathrm{ml}$ of $0.1 \mathrm{M}$ sodium borate buffer, $\mathrm{pH} 8.8$. The extract was centrifuged at $10,000 \times g$ for $20 \mathrm{~min}$ at $4^{\circ} \mathrm{C}$, and the supernatant was used as an enzyme source. The reaction mixture for PAL consisted of $10 \mathrm{mM}$ L-phenylalanine (Aldrich, Jerusalem, Israel), $0.1 \mathrm{M}$ borate buffer ( $\mathrm{pH} 8.8$ ), and $0.75 \mathrm{ml}$ of enzyme extract in a final volume of $2.5 \mathrm{ml}$. The reaction was incubated at $37^{\circ} \mathrm{C}$ for $60 \mathrm{~min}$ and terminated by the addition of $0.1 \mathrm{ml}$ of $5 \mathrm{~N} \mathrm{HCl}$. The product, cinnamic acid, was extracted in $3.5 \mathrm{ml}$ of diethyl ether and quantified by measuring the amount of trans-cinnamic acid formed at $269 \mathrm{~nm}(15)$.

Preparation of microsomes and isolation of plasma membranes. Plasma membranes were isolated from $40 \mathrm{~g}$ of exocarp strips after treatment with $0.2 \mathrm{mM} \mathrm{CaSO}_{4}$ for $24 \mathrm{~h}$. Plasma membranes were isolated at several periods after inoculation with $C$. gloeosporioides. Tissue was homogenized in a Waring blender at $4^{\circ} \mathrm{C}$, and microsomes were prepared as previously described (10).
The microsomes were suspended in a suspension medium $(330 \mathrm{mM}$ sucrose, $5 \mathrm{mM} \mathrm{KCl}$, and $5 \mathrm{mM}$ potassium phosphate buffer, $\mathrm{pH}$ 7.8), and the plasma membranes were separated by two-phase partitioning (13). The final composition of the phase system was $6.4 \%$ (wt/wt) dextran T500 and 6.4\% (wt/wt) polyethylene glycol (PEG 3350) in the suspension medium. The final upper phase was diluted 1:10 with a resuspension medium (330 mM sucrose, $1 \mathrm{mM}$ dithiothreitol, $5 \mathrm{mM} \mathrm{NaF}$, and $5 \mathrm{mM}$ MES-bis-tris propane, $\mathrm{pH}$ 7.8), and the purified plasma membranes were pelleted by centrifugation at 55,000 $\times \mathrm{g}$. The pellet was redissolved in $330 \mathrm{mM}$ sucrose, $5 \mathrm{mM}$ MES-bis-tris propane, and $1 \mathrm{mM}$ dithiothreitol adjusted to $\mathrm{pH} 7.0$ and brought to a concentration of 1 to $3 \mathrm{mg}$ protein $/ \mathrm{ml}$. Protein was assayed according to the Bradford method (6).

Reactive oxygen production measured by cytochrome $c$ reducing activity. The production of ROS was determined by cytochrome $c$ reduction dependent NADPH oxidation, as previously described (18). Reduction of the cytochrome $c$ molecule produces one molecule of reactive oxygen (16). The assay consisted of $20 \mu \mathrm{M}$ cytochrome $c$ (Sigma type VI), $100 \mu \mathrm{M}$ NADPH, $25 \mathrm{mM}$ potassium phosphate buffer ( $\mathrm{pH} 7.5)$, and $10 \mu \mathrm{g}$ of plasma membrane protein in a total volume of $1 \mathrm{ml}$. The reaction was initiated with the addition of NADPH, and the rate of cytochrome $c$ reduction was measured on a dual-wavelength spectrophotometer at $550 \mathrm{~nm}$, by the difference in the absence and presence of $100 \mu \mathrm{g}$ of superoxide dismutase, as described by Owen and Hancock (18).

$\mathbf{H}^{+}$-ATPase activity and rubidium uptake. Vanadate-sensitive plasma membrane was determined in the presence of $0.01 \%$ polyethylene 20 cetyl ether (Brij 58) and in the presence or absence of $0.2 \mathrm{mM} \mathrm{Na} \mathrm{VO}_{4}$. $\mathrm{H}^{+}$-ATPase activity was assayed as previously described (11), with variations: $4 \mu \mathrm{g}$ of plasma membrane protein was incubated for $30 \mathrm{~min}$ at $35^{\circ} \mathrm{C}$ in $0.5 \mathrm{ml}$ of a medium composed of $40 \mathrm{mM}$ MES-tris ( $\mathrm{pH} 6.5$ ), $0.25 \mathrm{M}$ sucrose, $100 \mathrm{mM} \mathrm{KCl}$, $3 \mathrm{mM} \mathrm{MgSO}_{4}$, and $3 \mathrm{mM}$ ATP. $\mathrm{H}^{+}$-ATPase activity in the sample plasma membrane was found to be $87 \%$ vanadate sensitive.

For rubidium uptake, exocarp tissue disks (about $5 \mathrm{~mm}$ in diameter and $1 \mathrm{~mm}$ thick, almost free of mesocarp) were prepared as described for microsome preparation. The disks were preincubated for $30 \mathrm{~min}$ in $0.2 \mathrm{mM} \mathrm{CaSO}_{4}$ at $25^{\circ} \mathrm{C}$. $\left[{ }^{86} \mathrm{Rb}\right] \mathrm{K}^{+}$was added to the vial for the last $20 \mathrm{~min}$ of the reaction. The uptake of $\left[{ }^{86} \mathrm{Rb}\right] \mathrm{K}^{+}$ into $70 \mathrm{mg}$ of avocado disk tissue (from five disks) was assayed in $5 \mathrm{ml}$ of $0.2 \mathrm{mM} \mathrm{CaSO}_{4}$ and $0.1 \mathrm{mM}\left[{ }^{86} \mathrm{Rb}\right] \mathrm{K}^{+}(700 \mathrm{~Bq} / \mu \mathrm{mol})$. Exchangeable was removed with three washings over a 5-min period in $15 \mathrm{ml}$ of ice-cold $10 \mathrm{mM} \mathrm{CaSO}_{4}$. The disks were transferred to vials for scintillation counting; $0.2 \mathrm{ml}$ of $1 \mathrm{~N} \mathrm{NaOH}$ was added for discoloration, and then $4 \mathrm{ml}$ of Opti-Fluor O (Packard, Groningen, The Netherlands) was added. Samples were counted in an MR 300 Lumitron Automatic liquid scintillation system (Packard).

Extraction and quantification of the antifungal diene. A 10-g sample of avocado exocarp (1-2 mm thick) was homogenized in 95\% ethanol in an Omni-Mixer (Sorvall, DuPont Company, Newtown, CT) at full speed for $3 \mathrm{~min}$. The ethanol extract was dried in a rotary evaporator at $40^{\circ} \mathrm{C}$ and then redissolved in $10 \mathrm{ml}$ of distilled water, and the organic phase was extracted by fractionation with dichloromethane. After two extractions, the organic phases were pooled, dried with anhydrous $\mathrm{MgSO}_{4}$ (Riedel-deHaen, Seelze, Germany), and evaporated to dryness. Samples were redissolved in $1 \mathrm{ml}$ of ethanol AR (Bio Lab, Jerusalem, Israel) and analyzed by HPLC (26). The average value of three separate extractions is presented. The experiment was repeated twice in each of two consecutive avocado seasons.

\section{RESULTS}

Phenotypic analysis of REMI mutants. Two C. gloeosporioides REMI mutant isolates, $\mathrm{Cg}-\mathrm{M}-142$ and $\mathrm{Cg}-\mathrm{M}-1150$, were compared to the wild-type (WT) isolate $\mathrm{Cg}$-14 for conidial germination, germ tube elongation, and appressorium formation on glass 
slides (Fig. 1A) and on avocado exocarp (Fig. 1B) 24 and $48 \mathrm{~h}$ after inoculation. Cg-M-142 and Cg-M-1150 were similar to the wild type in germination and germ tube elongation on the exocarp, but the two mutants were retarded on glass slides. Appressorium formation was delayed in Cg-M-142 and absent in Cg-M-1150 on both glass slides and exocarp. Appressorium formation by $\mathrm{Cg}-\mathrm{M}-$ 142 was similar to that of the WT within $48 \mathrm{~h}$ after inoculation, while Cg-M-1150 did not produce appressoria at all (Fig. 1A and B), even after $96 \mathrm{~h}$ (data not presented). Analysis of germ tube elongation showed that $\mathrm{Cg}-\mathrm{M}-142$ and $\mathrm{Cg}-\mathrm{M}-1150$ formed longer germ tubes on inoculated fruit than on glass slides (Fig. 1A and B).

Effect of preinoculation with REMI mutants on the development of decay. Inoculation of avocado fruit with Cg-M-142 increased the level of the antifungal diene approximately threefold
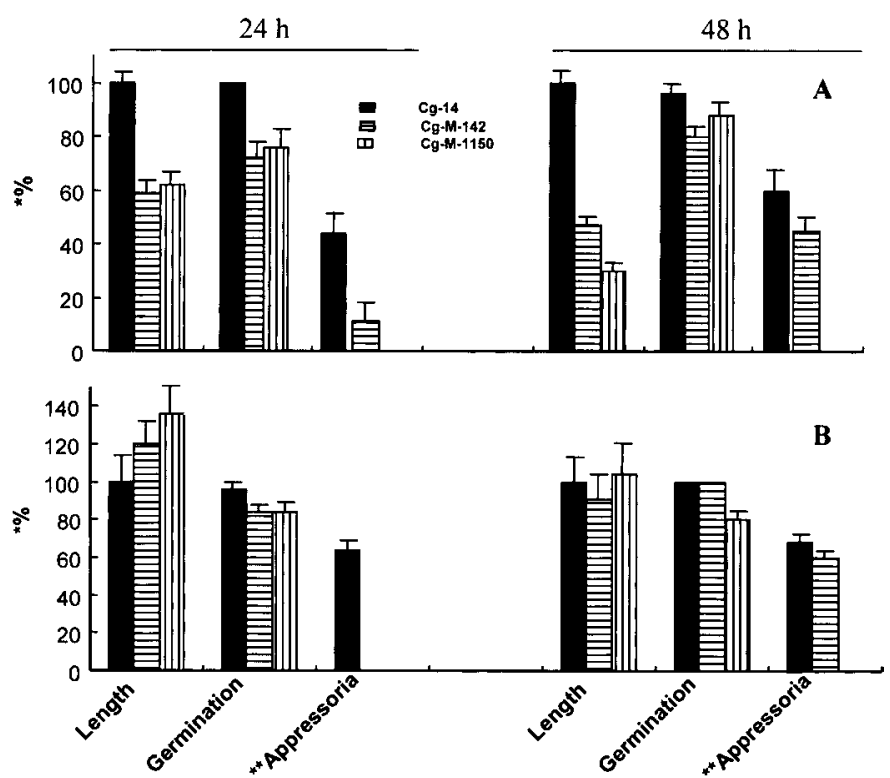

Fig. 1. Conidial germination, germ tube elongation, and appressorium formation by Colletotrichum gloeosporioides wild-type isolate $\mathrm{Cg}-14$ and the reduced-virulence mutants Cg-M-142 and Cg-M-1150 on glass slides A, and on exocarp of avocado fruit of the cultivar Fuerte B, 24 and $48 \mathrm{~h}$ after inoculation. The data and standard errors are from three replications. *Percentages relative to the wild type. **Percentage of germlings that formed appressoria.

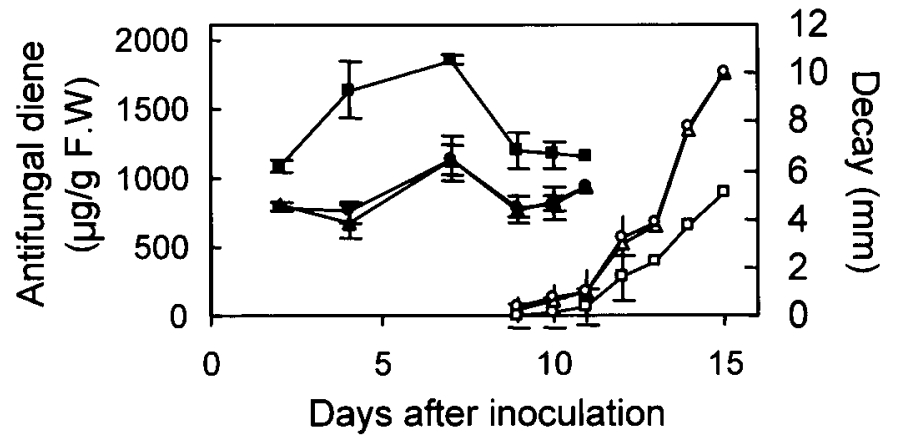

Fig. 2. Development of decay caused by Colletotrichum gloeosporioides isolate $\mathrm{Cg}-14$ in avocado fruit of the cultivar Fuerte following preinoculation with the reduced-virulence mutants Cg-M-142 (ם) and Cg-M-1150 (•) or with water as a control $(\boldsymbol{\Delta})$. Ten freshly harvested fruits were dipped for $30 \mathrm{~s}$ in a conidial suspension of Cg-M-142 or Cg-M-1150 or in water, inoculated $24 \mathrm{~h}$ later at six points with the wild-type $\mathrm{Cg}-14$, and incubated for 15 days. The effect of the reduced-virulence mutants on the antifungal diene was done in parallel by inoculation of the whole fruit with spores of the mutant isolates or with water treatment only. Levels of diene (filled symbols) and decay development (open symbols) were recorded. The experiments were repeated three times in two consecutive harvesting seasons. The data and standard errors are from one representative experiment. within 4 days (Fig. 2). The diene level remained higher in treated fruit even 11 days after inoculation with Cg-M-142. Decay symptoms were delayed by 2 days in fruit preinoculated with $\mathrm{Cg}-\mathrm{M}$ 142 , compared to the control fruit and fruit preinoculated with $\mathrm{Cg}$ $\mathrm{M}-1150$. Diene levels were similar in fruit inoculated with $\mathrm{Cg}-\mathrm{M}-$ 1150 and the control fruit, during the monitored period. In addition, the development of symptoms in fruit preinoculated with $\mathrm{Cg}$ M-1150 was similar to that in control fruit (Fig. 2).

$\mathrm{H}^{+}$-ATPase activity, rubidium uptake, and $\mathrm{O}_{2}$ generationdependent NADPH oxidation. Exocarp slices inoculated with the mutant strains were tested for early signaling affects. $\mathrm{H}^{+}$-ATPase activity in the plasma membrane extracted from exocarp inoculated with Cg-M-142 increased fourfold within $2 \mathrm{~h}$ after inoculation (Fig. 3A) and $2 \mathrm{~h}$ later dropped to the same level as the control. Inoculation with $\mathrm{Cg}-\mathrm{M}-1150$, however, had no affect on $\mathrm{H}^{+}$-ATPase activity $2 \mathrm{~h}$ after treatment, with a small increase $4 \mathrm{~h}$ after treatment. This observation was further confirmed by monitoring $\mathrm{Rb}^{+}$uptake, which showed a pattern similar to that of $\mathrm{H}^{+}$-
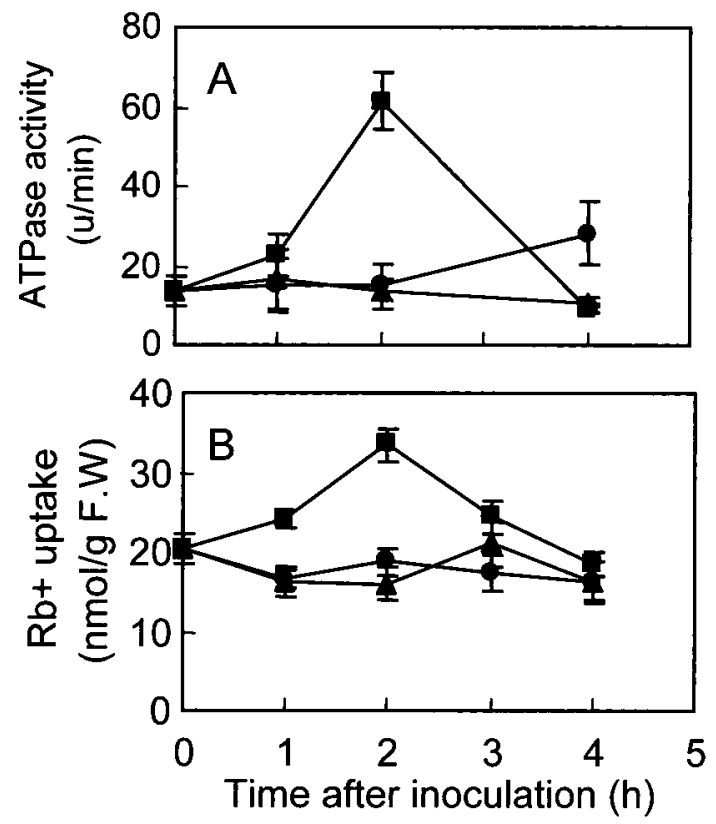

Fig. 3. Effect of preinoculation of avocado fruit exocarp with Colletotrichum gloeosporioides mutant isolates Cg-M-142 (অ) and Cg-M-1150 (•) or with water $(\boldsymbol{\Lambda})$ on the induction of plasma membrane $\mathrm{H}^{+}$-ATPase $\mathbf{A}$, and rubidium uptake B, on extracted plasma membranes. The bars represent standard error. The data from three experiments with five replications per treatment were pooled. The experiments were repeated in two consecutive avocado seasons.

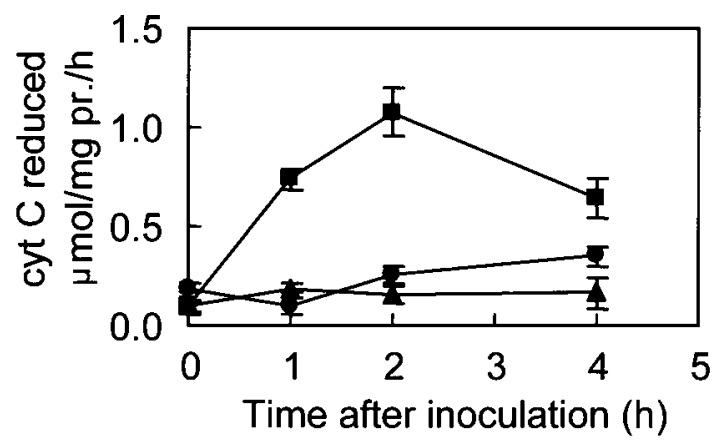

Fig. 4. Time course of the production of reactive oxygen species in plasma membranes isolated from avocado fruit exocarp inoculated with Colletotrichum gloeosporioides mutant isolates Cg-M-142 (ם) and Cg-M-1150 (•) or with water $(\mathbf{A})$. The bars represent standard error from one representative experiment run four times with three replications. The experiment was repeated in two consecutive avocado seasons. 
ATPase activity, while Cg-M-1150 followed the same pattern as the control (Fig. 3B). A transient sixfold increase in NADPH oxidase activity of exocarp inoculated with $\mathrm{Cg}-\mathrm{M}-142$ occurred 1 to $2 \mathrm{~h}$ after inoculation (Fig. 4). In exocarp inoculated with $\mathrm{Cg}-\mathrm{M}$ 1150 , cytochrome $c$ reduction was unaffected $2 \mathrm{~h}$ after inoculation and a increased slightly $4 \mathrm{~h}$ after inoculation (Fig. 4).

Induction of PAL and epicatechin level. Inoculation of exocarp slices with Cg-M-142 increased PAL activity more than twofold within $10 \mathrm{~h}$ (Fig. 5A), whereas PAL activity was reduced in exocarp inoculated with $\mathrm{Cg}-\mathrm{M}-1150$, compared to the control. Epicatechin also increased twofold in exocarp inoculated with $\mathrm{Cg}$ M-142, whereas no change in epicatechin levels was found after inoculation with Cg-M-1150 (Fig. 5B). Epicatechin levels increased when whole avocado fruit was preinoculated with $\mathrm{Cg}-\mathrm{M}$ 142 or Cg-M-1150. However, only the epicatechin increase in fruit inoculated with Cg-M-142 was significant (a twofold increase within $120 \mathrm{~h}$ after inoculation) (Fig. 6), while no significant increase was found in fruit inoculated with Cg-M-1150.

\section{DISCUSSION}

Development of disease caused by pathogens in the genus Colletotrichum requires adhesion of conidia to the surface of host tissue, followed by conidial germination and the formation of appressoria to penetrate the host tissue $(3,19)$. Two reduced-pathogenicity REMI mutants, Cg-M-142 and Cg-M-1150 (36), did not differ from the wild-type $C$. gloeosporioides in germination rate or germ tube elongation on fruit exocarp. The most striking differences between the two mutants and the wild type were (i) the lack of appressorium formation by $\mathrm{Cg}-\mathrm{M}-1150$ and the delayed appressorium formation by $\mathrm{Cg}-\mathrm{M}-142$ and (ii) the capability of Cg-M-142 to induce resistance to the wild-type $C$. gloeosporioides isolate. The importance of appressoria in fungal attack has been extensively studied $(30,37)$; however, it has not been examined for its importance in triggering signals leading to increased resistance.
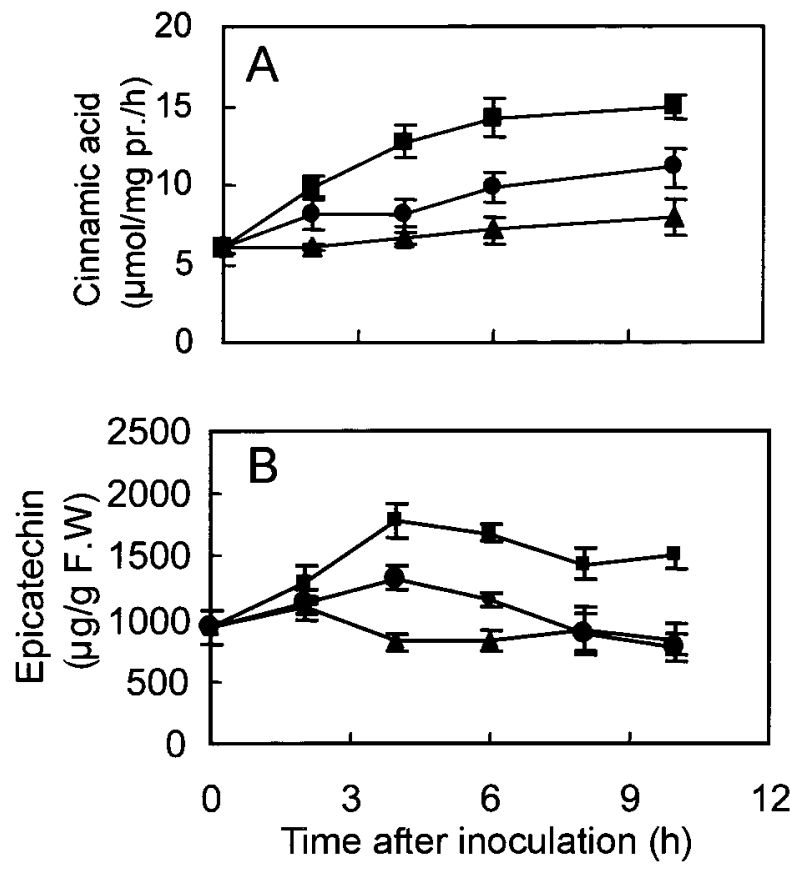

Fig. 5. Phenylalanine ammonia lyase activity $\mathbf{A}$, and epicatechin $\mathbf{B}$, in slices of avocado fruit exocarp inoculated with Colletotrichum gloeosporioides

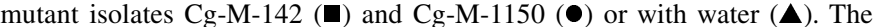
bars represent standard error. The data from three experiments with five replications per treatment were pooled. The experiments were repeated in two consecutive avocado seasons.
Avocado fruit exocarp, like other plant organs $(7,9)$, recognizes penetrating hyphae of wild-type $C$. gloeosporioides appressoria at the level of the host plasma membrane (4). This recognition occurs by the release of soluble cell wall sugars, probably sensed by a heterotrimeric G-protein receptor that activates a G-protein (32, 33) that transduces the signal by activating one or more membrane-bound phosphates, resulting in the dephosphorylation of the host plasma membrane $\mathrm{H}^{+}$-ATPase and activation of NADPH oxidase (4) (Fig. 7). We cannot exclude the presence of other receptors in the plasma membrane that may sense the penetrating fungus as well. However, activation of $\mathrm{H}^{+}$-ATPase and $\mathrm{H}_{2} \mathrm{O}_{2}$ production was stimulated by the appressorium-forming isolate $\mathrm{Cg}$ $\mathrm{M}-142$ and not by the non-appressorium-forming isolate $\mathrm{Cg}-\mathrm{M}$ 1150. The latter caused a marginal delayed increase in both signals, had no effect on rubidium uptake, and failed to alter fruit resistance. The lack of induction of resistance indicates the importance of appressorium formation in initiating defense signals, such as the proton electrochemical gradient across the plasma membrane (28). In avocado fruit, $\mathrm{H}^{+}$-ATPase was induced by the penetrating appressoria, along with $\mathrm{Rb}^{+}$uptake, confirming the opening of $\mathrm{K}^{+}$channels as an early reaction to fungal invasion. ROS production has been shown to be an early response in plant pathogen interactions in general $(1,17)$ and in avocado fruit $(4)$. Inoculation of avocado exocarp tissue with the wild-type Cg-14 increased ROS production threefold within $2 \mathrm{~h}$, while inoculation with Cg-M-142 increased ROS production sixfold, which can explain its increased resistance effect. Cg-M-1150 did not significantly affect ROS levels. In addition, the application of $\mathrm{Ca}^{+2}$ ionophore (A-23187), which opens $\mathrm{Ca}^{+2}$ channels, stimulated the production of $\mathrm{H}_{2} \mathrm{O}_{2}$ (4) (Fig. 7). Thus the level of $\mathrm{H}_{2} \mathrm{O}_{2}$ in the fruit may predict the level of fruit resistance against fungal attack. In addition to these responses, $\mathrm{Cg}-\mathrm{M}-142$ activated the resistancerelated phenylpropanoid pathway, where PAL activity was higher and epicatechin accumulation was 30\% higher than that induced by the wild-type $\mathrm{Cg}-14$ (4). Moreover, in vivo defense experiments on whole fruit showed a threefold increase in epicatechin production in fruit inoculated with $\mathrm{Cg}-\mathrm{M}-142$, compared to the control. Activation of the phenylpropanoid pathway increases the production of epicatechin, an antioxidant, which protects the reduction of the antifungal diene by inhibiting lipoxygenase (2). Epicatechin has also been found to inhibit $C$. gloeosporioides polygalacturonase and pectate lyase $(34,35)$ (Fig. 7$)$, and the latter was recently found to be an important virulence factor in fruit-fungus interactions (35). The induction of PAL activity by $\mathrm{H}_{2} \mathrm{O}_{2}$ in avocado fruit is in agreement with the results of studies of Arabidopsis and tobacco cell cultures, in which the addition of exogenous $\mathrm{H}_{2} \mathrm{O}_{2}$ triggered PAL expression $(14,17)$. This suggests that PAL activity is stimulated after direct penetration by appressoria, as was observed in barley leaves after inoculation with Erysiphe graminis

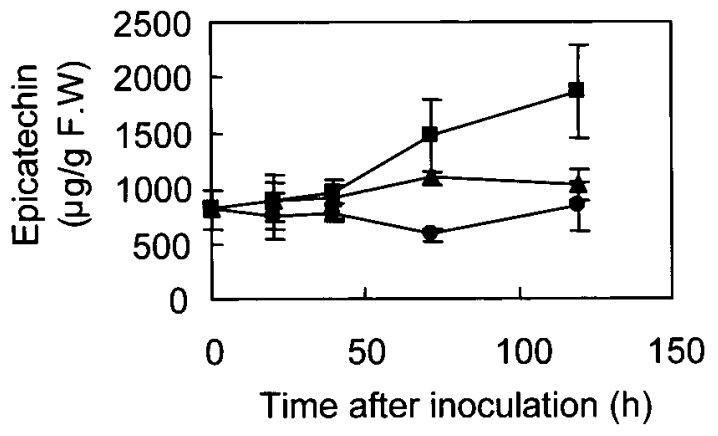

Fig. 6. Epicatechin in avocado fruit exocarp inoculated with Colletotrichum gloeosporioides mutant isolates Cg-M-142 (ם) and Cg-M-1150 (•) or with water $(\boldsymbol{\Delta})$. The bars represent standard error in one representative experiment run four times with three replications. The experiment was repeated in two consecutive avocado seasons. 


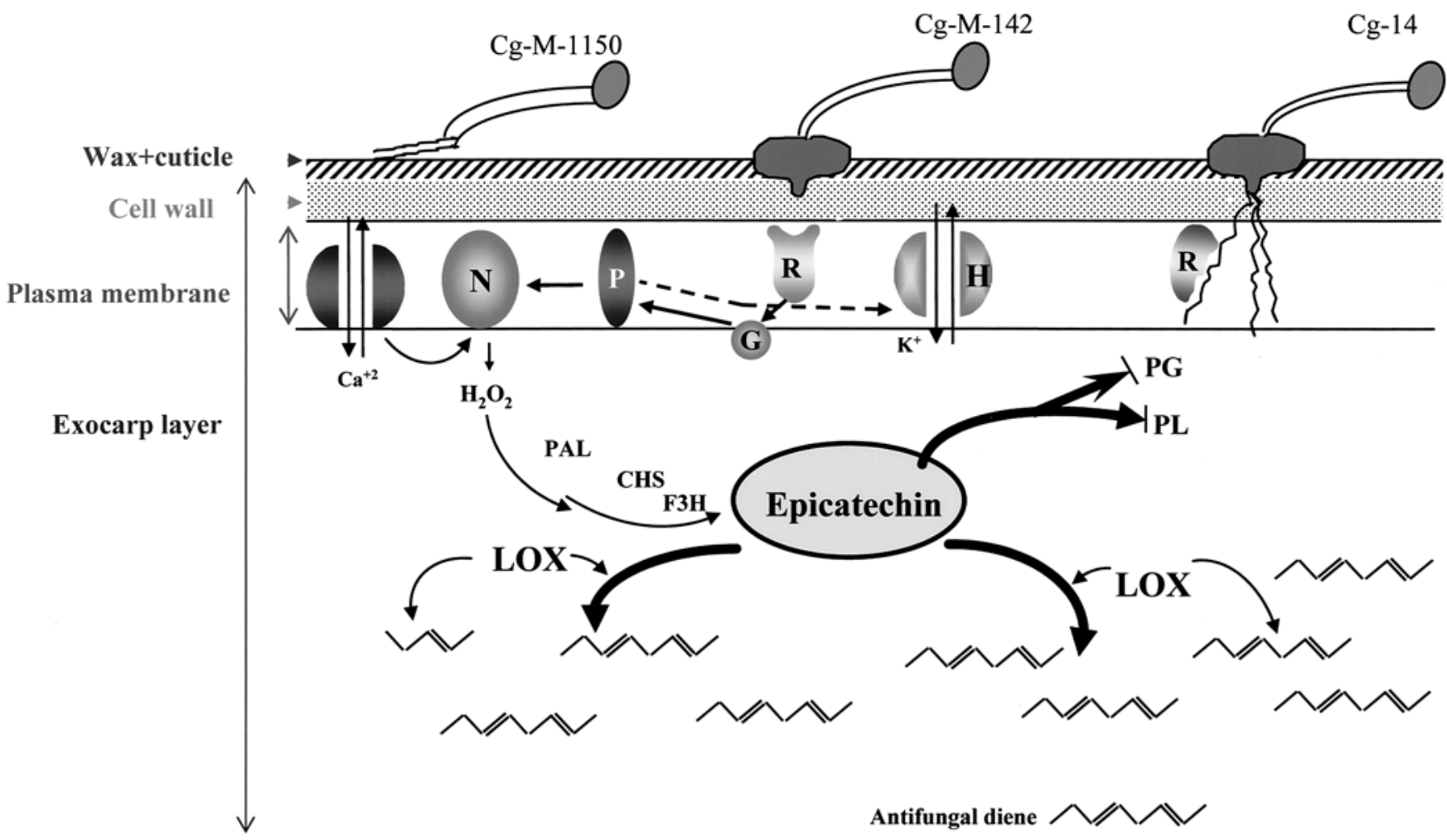

Fig. 7. General scheme of proposed interaction between fruit of the avocado cultivar Fuerte and Colletotrichum gloeosporioides isolates Cg-14, Cg-M-142, and Cg-M-1150, during the attack/protection actions of the different strains. The scheme was generated from our data and data published in other fungal systems as cited in the text. $\mathrm{N}=\mathrm{NADPH}$-oxidase. $\mathrm{P}=$ phosphates. $\mathrm{G}=$ heterotrimeric $\mathrm{G}$-protein. $\mathrm{H}=\mathrm{H}^{+}$-ATPase. $\mathrm{R}=$ receptor. Vertical arrows $=$ ion channels. $\mathrm{PAL}=$ phenylalanine ammonia lyase. $\mathrm{CHS}=$ chalcon synthase. $\mathrm{F} 3 \mathrm{H}=$ flavonone-3-hydroxylase. $\mathrm{LOX}=$ lipoxygenase. $\mathrm{PG}=$ polygalacturonase $. \mathrm{PL}=$ pectate lyase.

or E. pisi (29), via the production of $\mathrm{H}_{2} \mathrm{O}_{2}$. Although Cg-M-1150 showed a marginal increase in early signals as well as in PAL activity, it did not have any effect on fruit resistance. It is possible that compounds secreted by the fungus during its growth on the exocarp generate this effect. Since PAL is the first enzyme in the phenylpropanoid pathway that branches into various routes, the increase in PAL may affect other downstream products unrelated to resistance.

The genetic differences between the wild-type $\mathrm{Cg}$-14 and its derived isolate $\mathrm{Cg}-\mathrm{M}-142$ in their ability to induce resistance has not yet been elucidated. Several genes have been reported to affect appressorium formation (37). A nonpathogenic C. lagenarium mutant in mitogen-activated protein (MAP) kinase, CMK1 gene, showed reduced conidiation and failed to form appressoria (30). The disruption of Botrytis cinerea BMP1, a gene encoding MAPkinase, prevented appressorium formation and pathogenicity (38). Yang and Dickman (37) showed that strains of $C$. trifolii deficient in protein-kinase A were unable to infect intact alfalfa plants, likely because of a failure to penetrate. $\mathrm{Cg}-\mathrm{M}-142$ has been shown to harbor a single REMI integration site, which suggests a single gene disruption, whereas two independent integration sites have been found in Cg-M-1150, possibly disrupting one or two genes to cause its unique phenotype (36). The genes disrupted by REMI technology in both mutants are currently under investigation. Although the flanking regions have been cloned, we did not find any homology in the known published genetic data.

The two REMI mutant isolates allowed us to demonstrate the importance of appressorium formation dynamics in triggering fruit resistance. In general appearance, the fruit inoculated with $\mathrm{Cg}-\mathrm{M}$ 142 was less decayed than fruit inoculated with Cg-M-1150 or with the control (data not shown). This observation indicates that early signaling by $\mathrm{Cg}-\mathrm{M}-142$ had succeeded in elevating resistance in fruit naturally infected with C. gloeosporioides as well. We suggest that the induction of fruit resistance is a result of greater epicatechin accumulation, derived from early signals generated by delayed appressorium formation and invading Cg-M142 hyphae, in contrast to the fast penetration by the wild-type isolate $(35,36)$. This fast penetration by the wild-type isolate has generated a fast and transient activation of resistance mechanism, but this effect did not delay symptom development in ripening fruit (20). Lack of appressorium formation by the Cg-M-1150 mutant is assumed to be the reason for the lack of induction of resistance and failure to colonize the fruit.

\section{ACKNOWLEDGMENTS}

We thank R. Ardi for drawing the initial general scheme model. This research was supported by Research Grants IS-2686-96 to D. Prusky from BARD, the U.S. Binational Agricultural Research and Development Fund, the German-Israel Agricultural Research Agreement (GIARA) for the Benefit of the Third World, and the U.S.-Israel Cooperative Development Research (CDR) Program. The first author, N. Yakoby, was granted personal scholarships from the Israel Fruit Marketing Board, Ministry of Agriculture and Rural Development, and from the Association of Trustees and Friends of the Agricultural Research Organization.

\section{LITERATURE CITED}

1. Apostol, I., Hienstein, P. F., and Low, P. S. 1989. Rapid stimulation of an oxidative burst during elicitation of cultured plant cells. Plant Physiol. 90:109-116.

2. Ardi, R., Kobiler, I., Keen, N. T., and Prusky, D. 1998. Involvement of epicatechin biosynthesis in the resistance of avocado fruits to postharvest decay. Physiol. Mol. Plant Pathol. 53:269-285.

3. Baily, J. A., O'Connel, R. J., Pring, R. J., and Nash, C. 1992. Infection strategies of Colletotrichum species. Pages 88-120 in: Colletotrichum: Biology, Pathology, and Control. J. A. Baily and M. J. Jeger, eds. CAB International, Wallingford, U.K.

4. Beno-Moualem, D., and Prusky, D. 2000. Early events during quiescent infection development by Colletotrichum gloeosporioides in unripe avocado fruits. Phytopathology 90:553-559. 
5. Binyamini, N., and Schiffmann-Nadel, M. 1972. Latent infection in avocado fruit due to Colletotrichum gloeosporioides. Phytopathology 62: 592-594.

6. Bradford, M. M. 1976. A rapid and sensitive method for the quantitation of microgram quantities of protein utilizing the principle of protein-dye binding. Anal. Biochem. 72:248-254.

7. Cheong, J. J., and Hahn, M. 1991. A specific high-affinity binding site for the hepta-b-glucoside elicitor exists in soybean membranes. Plant Cell 3:137-147.

8. Coates, L. M., Muirhead, I. F., Irwin, J. A. G., and Gowanlock, D. 1993. Initial infection processes by Colletotrichum gloeosporioides on avocado fruit. Mycol. Res. 97:1363-1370.

9. Cosio, E. G., Frey, T., and Ebel, J. 1990. Solubilization and characterization of the binding sites for fungal b-glucans from soybean cell membranes. FEBS Lett. 264:235-238.

10. Giannini, J. L., Gildensoph, L. H., and Briskin, D. P. 1987. Selective production of sealed plasmamembrane vesicles from red beet (Beta vulgaris L.) storage tissue. Arch. Biochem. Biophys. 254:621-630.

11. Jacoby, B., and Rudich, B. 1987. Compound 48/80, a calmodulin antagonist, inhibits ion-porter function in plant roots. Physiol. Plant 70: 617-621

12. Karni, L., Prusky, D., Kobiler, I., Bar-Shira, E., and Kobiler, D. 1989. Involvement of epicatechin in the regulation of lipoxygenase activity during activation of quiescent Colletotrichum gloeosporioides infections of ripening avocado fruits. Physiol. Mol. Plant Pathol. 35:367-374.

13. Larsson, C., Widell, S., and Kjellbom, P. 1987. Preparation of high purity plasma membranes. Methods Enzymol. 148:558-568.

14. Levine, A., Tenhaken, R., Dixon, R., and Lamb, C. $1994 . \mathrm{H}_{2} \mathrm{O}_{2}$ from the oxidative burst orchestrates the plant hypersensitive disease resistance response. Cell 79:583-593.

15. Lisker, N., Cohen, L., Chalutz, E., and Fuchs, Y. 1983. Fungal infection suppress ethylene induced PAL activity in grapefruit. Physiol. Plant Pathol. 22:331-338.

16. Mayo, L. A., and Curnutte, J. T. 1990. Kinetic microplate assay for superoxide production by neutrophils and other phagocytic cells. Methods Enzymol. 186:567-575.

17. Mehdy, C. N. 1994. Active oxygen species in plants defense against pathogens. Plant Physiol. 105:467-472.

18. Owen, T. G. J., and Hancock, J. T. 1994. Assays of plasma membrane NADPH oxidase. Methods Enzymol. 186:222-229.

19. Prusky, D. 1996. Pathogen quiescence in postharvest diseases. Annu. Rev. Phytopathol. 34:413-434.

20. Prusky, D., Freeman, S., Rodriguez, R. J., and Keen, N. T. 1994. A nonpathogenic mutant strain of Colletotrichum magna induces resistance to C. gloeosporioides in avocado fruits. Mol. Plant-Microbe Interact. 7: 326-333.

21. Prusky, D., Hamdan, H., Ardi, R., and Keen, N. T. 1996. Induction of biosynthesis of the epicatechin in avocado suspension cells treated with an enriched $\mathrm{CO}_{2}$ atmosphere. Physiol. Mol. Plant Pathol. 48:171-178.

22. Prusky, D., and Keen, N. T. 1993. Involvement of preformed antifungal compounds in the resistance of subtropical fruits to fungal decay. Plant Dis. 7:114-119.

23. Prusky, D., Keen, N. T., and Eaks, I. 1983. Further evidence for the involvement of a preformed antifungal compounds in the latency of Colletotrichum gloeosporioides on the unripe fruits. Physiol. Plant Pathol. 22:
189-198.

24. Prusky, D., Keen, N. T., Sims, J. J., and Midland, S. L. 1982. Possible involvement of an antifungal diene in the latency of Colletotrichum gloeosporioides on unripe avocado fruits. Phytopathology 72:1578-1582.

25. Prusky, D., and Plumbley, R. A. 1992. Quiescent infection of Colletotrichum in tropical and subtropical fruits. Pages 289-307 in: Colletotrichum: Biology and Control. J. A. Baily and M. Jeger, eds. CAB International, Wallingford, U.K.

26. Prusky, D., Plumbley, R. A., and Kobiler, I. 1991. The relationship between antifungal diene levels and fungal inhibition during quiescent infection of unripe avocado fruits by Colletotrichum gloeosporioides. Plant Pathol. 40:45-52.

27. Redman, R. S., Freeman, S., Clifton, D. R., Morrel, J., Brown, G., and Rodriguez, R. J. 1999. Biochemical analysis of plant protection afforded by a nonpathogenic endophytic mutant of Colletotrichum magna. Plant Physiol. 119:795-804

28. Schaller, A., and Oecking, C. 1999. Modulation of plasma membrane $\mathrm{H}^{+}$-ATPase activity differentially activates wound and pathogen defense responses in Tomato plants. Plant Cell 11:263-272.

29. Shiraishi, T., Yamada, T., Nicholson, L., and Kunoh, H. 1995. Phenylalanine ammonia-lyase in barley: Activity enhancement in response to Erysiphe graminis hordi (race 1) a pathogen, and Erysiphe pisi, a nonpathogen. Physiol. Mol. Pathol. 46:153-162.

30. Takano, Y., Kikuchi, T., Kubo, Y., Hamer, J. E., Mise, K., and Furusawa, I. 1999. The MAP kinase gene CMK1 of Colletotrichum lagenarium regulates germination, appressorium formation, and invasive growth. (Abstr.) Int. Soc. Mol. Plant-Microbe Interact. Int. Congr. 9th.

31. Tu, J. C. 1985. An improved Mathur's medium for growth, sporulation and germination of conidias of Colletotrichum lindemuthianum. Microbiosis 44:87-93.

32. Vera-Estrella, R., Barkla, B. J., Higgins, V. J., and Blumwald, E. 1994 Plant defense response to fungal pathogens: Activation of host plasma membrane $\mathrm{H}^{+}$-ATPase by elicitor-induced enzyme dephosphorylation. Plant Physiol. 104:209-215.

33. Vera-Estrella, R., Barkla, B. J., Higgins, V. J., and Blumwald, E. 1994. Plant defense response to fungal pathogens: G-protein-mediated changes in host plasmamembrane redox reactions. Plant Physiol. 106:97-102.

34. Wattad, C., Dinoor, A., and Prusky, D. 1994. Purification of pectate lyase produced by Colletotrichum gloeosporioides and its inhibition by epicatechin: A possible factor involved in the resistance of unripe avocado fruits to anthracnose. Mol. Plant-Microbe Interact. 7:293-297.

35. Yakoby, N., Beno-Moualem, D., Keen, N. T., Dinoor, A., Pines, O., and Prusky, D. 2001. Colletotrichum gloeosporioides pel $\mathrm{B}$ is an important virulence factor in avocado fruit-fungus interaction. Mol. Plant-Microbe Interact. 14:988-995.

36. Yakoby, N., Zhou, R., Kobiler. I., Dinoor, A., and Prusky, D. 2001. Development of Colletotrichum gloeosporioides restriction enzymemediated integration mutants as biocontrol agents against anthracnose disease in avocado fruit. Phytopathology 91:143-148.

37. Yang, Z., and Dickman, M. B. 1999. Colletotrichum trifolii mutants disrupted in the catalytic subunit of cAMP-dependent protein kinase are nonpathogenic. Mol. Plant-Microbe Interact. 12:430-439.

38. Zheng, L., Campbell, M., Murphy, J., Lam, S., and Xu, J. R. 2000. The $B M P 1$ gene is essential for pathogenicity in the gray mold fungus Botrytis cinerea. Mol. Plant-Microbe Interact. 13:724-732. 